\title{
THE EXTENSION OF REGULAR HOLOMORPHIC MAPS ${ }^{1}$
}

\author{
H. L. ROYDEN
}

\begin{abstract}
Let $f$ be a holomorphic map of a $k$-dimensional polydisk in $C^{n}$ into an $n$-dimensional complex manifold $M$ which is regular at the origin. It is shown that $f$ can be extended to a neighborhood in $\boldsymbol{C}^{n}$ of a slightly smaller polydisk so that the extension is also regular. If $f$ is an embedding or immersion, then the extension may also be taken to be an embedding or immersion.
\end{abstract}

Let $\Delta^{k}$ be the unit polydisk in $C^{k}$, and let $\Delta_{r}^{k}$ denote the polydisk of radius $r$. Suppose that $f$ is a holomorphic map of $\Delta^{k}$ into an $n$-dimensional complex manifold $M$, and suppose that $f$ is regular at 0 ; i.e., its Jacobian matrix has rank $k$ there. In the infinitesimal theory of the Kobayashi metric (cf. [4]), it is important to know that $f$ can be extended to a map $F$, defined on a neighborhood $\Delta_{r}^{k} \times \Delta^{n-k}$ in $C^{n}$ of a slightly smaller polydisk $\Delta_{r}^{k}$, so that $F$ is also regular at 0 . It is the purpose of this note to establish this result (Proposition 2) and to show that $F$ may be taken to be an embedding or immersion provided $f$ is (Propositions 1 and 3).

These results are not very difficult if $M$ is a Stein manifold, and the major effort in the proof is to show that we can reduce the problem to that case. To do this, we construct, in the case of an embedding, an open subset $O$ of $M$, which contains $f\left[\bar{\Delta}_{r}^{k}\right]$ and is Stein.

The proofs given here extend to the case when $\Delta^{k}$ is replaced by any contractible domain of holomorphy $D$ in $C^{k}$ and $\bar{\Delta}_{r}^{k}$ by any compact subset of $D$. Chester Seabury has extended these results to the case when $\Delta^{k}$ is replaced by any contractible Stein manifold, and has generalizations for the case of arbitrary Stein manifolds.

We begin by considering the case when $f$ is an embedding:

PROPOSITION 1. Let $f$ be a holomorphic embedding of the unit polydisk $\Delta^{k}$ into an $n$-dimensional manifold $M$. Then, given $r<1$, there is a holomorphic embedding $F$ of $\Delta^{k} \times \Delta^{n-k}$ into $M$ such that $F=f$ on $\Delta^{k} \times\{0\}$.

Received by the editors February 26, 1973 and, in revised form, June 6, 1973.

AMS (MOS) subject classifications (1970). Primary 32H99, 32C10.

Key words and phrases. Extension of holomorphic maps, holomorphic immersion, holomorphic embedding, equi-dimensional holomorphic maps.

${ }^{1}$ This work was partially supported by NSF Grant GP 33942X. 
The function $\phi$ defined in $\Delta^{k}$ by setting

$$
\phi(z)=c \sum\left|z_{i}\right|^{2}\left(1-\left|z_{i}\right|^{2}\right)^{-1}
$$

is, for $c>0$, a convex function tending to infinity as $z$ approaches the boundary of $\Delta^{k}$. Thus, for a suitable choice of $c$, the region $D=\{z: \phi(z)<1\}$ is convex, with $\bar{\Delta}_{r}^{k} \subset D$ and $\bar{D} \subset \Delta^{k}$. Choose an $r^{\prime}<1$, so that $\bar{D} \subset \Delta_{r^{\prime}}^{k}$, and denote $\Delta_{r^{\prime}}^{k}$ by $\Delta^{\prime}$.

Since $f$ is an embedding and $f\left[\bar{\Delta}^{\prime}\right]$ compact, we may choose for each point $a \in \Delta^{\prime}$ a coordinate neighborhood $U_{\alpha}$ of $f(a)$ with coordinates $z_{\alpha}^{1}, \cdots, z_{\alpha}^{k}, w_{\alpha}^{k+1}, \cdots, w_{\alpha}^{n}$ such that $U_{\alpha}=\left\{\left\langle z_{\alpha}, w_{\alpha}\right\rangle:\left|z_{\alpha}^{i}-a^{i}\right|<\varepsilon,\left|w_{\alpha}^{j}\right|<\delta\right\}$, and such that, on $V_{\alpha}=f^{-1}\left[U_{\alpha}\right] \cap \Delta^{\prime}$, the map $f$ is given by $f(z)=\langle z, 0\rangle$. We also assume $\bar{U}_{\alpha}$ is compact and contained in an open set where the coordinates are still valid. Such coordinates and coordinate neighborhoods are said to be admissible, and we will use only such coordinates in the sequel. For convenience of notation, we identify $\Delta^{\prime}$ with its image under $f$, and the coordinates $z$ with $z_{\alpha}$ when $w_{\alpha}=0$. Thus $V_{\alpha}=U_{\alpha} \cap \Delta^{\prime}$.

Cover $\bar{\Delta}^{\prime}$ by a finite collection $\left\{U_{\alpha}\right\}$ of admissible coordinate neighborhoods. On $V_{\alpha} \cap V_{\beta}$, the Jacobian matrix of the coordinates $\left\langle z_{\alpha}, w_{\alpha}\right\rangle$ has the form

$$
\left[\begin{array}{cc}
I & A_{\alpha \beta}(z) \\
0 & B_{\alpha \beta}(z)
\end{array}\right]
$$

where the $B_{\alpha \beta}$ are holomorphic matrices satisfying $B_{\alpha \beta}=B_{\beta \alpha}^{-1}$ and $B_{\alpha \gamma}=B_{\alpha \beta} B_{\beta \gamma}$. Thus the holomorphic matrices $B_{\alpha \beta}(z)$ define a holomorphic vector bundle over $\Delta^{\prime}$ (in fact, the normal bundle of the embedding). Since $\Delta^{\prime}$ is contractible and Stein, a theorem of Grauert [2, Satz 5] asserts that this bundle is holomorphically trivial. Hence there are invertible holomorphic matrices $B_{\alpha}(z)$ in $V_{\alpha}$, such that $B_{\alpha \beta}=B_{\alpha} B_{\beta}^{-1}$. (Strictly speaking, we may have to pass to a refinement of the covering $\left\{V_{\alpha}\right\}$, but we again call this refinement $\left\{V_{\alpha}\right\}$ and choose corresponding $U_{\alpha}$.) In $U_{\alpha}$, we introduce new coordinates by setting $\tilde{z}_{\alpha}=z_{\alpha}$ and $\tilde{w}_{\alpha}=B_{\alpha}^{-1}\left(z_{\alpha}\right) w_{\alpha}$. Then on $V_{\alpha} \cap V_{\beta}$, the Jacobian matrix of the new coordinates has the form

$$
\left[\begin{array}{cc}
I & A_{\alpha \beta}(z) \\
0 & I
\end{array}\right] .
$$

The matrices $A_{\alpha \beta}$ now satisfy $A_{\alpha \beta}=-A_{\beta \alpha}$ and $A_{\alpha \beta}+A_{\beta \gamma}=A_{\alpha \gamma}$. Thus they determine a one-dimensional cocycle on $\left\{V_{\alpha}\right\}$ with coefficients in the sheaf $\mathcal{O}_{\Delta}^{a}$, with $q=k(n-k)$. Since $H^{1}\left(\Delta^{\prime}, \mathcal{O}^{q}\right)=0$, we may, possibly after passing to a refinement of $\left\{V_{\alpha}\right\}$, choose holomorphic matrices $A_{\alpha}(z)$ in $V_{\alpha}$, so that $A_{\alpha \beta}=A_{\alpha}-A_{\beta}$. In $U_{\alpha}$, we define new functions by setting $\hat{z}_{\alpha}=z_{\alpha}-A_{\alpha}\left(z_{\alpha}\right) \tilde{w}_{\alpha}$ and $\hat{w}_{\alpha}=\tilde{w}_{\alpha}$. Since the Jacobian of $\left\langle\hat{z}_{\alpha}, \hat{w}_{\alpha}\right\rangle$ with respect 
to $\left\langle z_{\alpha}, w_{\alpha}\right\rangle$ is nonzero on $\bar{V}_{\alpha}$, and the mapping $\left\langle z_{\alpha}, w_{\alpha}\right\rangle \rightarrow\left\langle\hat{z}_{\alpha}, \hat{w}_{\alpha}\right\rangle$ is the identity on $\bar{V}_{\alpha}$, it follows that $\left\langle\hat{z}_{\alpha}, \hat{w}_{\alpha}\right\rangle$ are coordinates in some neighborhood of $\bar{V}_{\alpha}$. Since these are admissible coordinates, we have established the following lemma:

Lemma 1. It is possible to choose admissible coordinates $\left\langle z_{\alpha}, w_{\alpha}\right\rangle$ in neighborhoods $U_{\alpha}$ covering $\Delta^{\prime}$ so that, on $U_{\alpha} \cap U_{\beta} \cap \Delta^{\prime}$, the Jacobian matrix of $\left\langle z_{\alpha}, w_{\alpha}\right\rangle$ with respect to $\left\langle z_{\beta}, w_{\beta}\right\rangle$ is the identity.

We say that the coordinates $\left\langle z_{\alpha}, w_{\alpha}\right\rangle$ and $\left\langle z_{\beta}, w_{\beta}\right\rangle$ match to order $l$ if, in $U_{\alpha} \cap U_{\beta}$, we have $z_{\alpha}=z_{\beta}+$ terms of order $l+1$ in $w_{\beta}$, and $w_{\alpha}=w_{\beta}+$ terms of order $l+1$ in $w_{\beta}$.

Lemma 2. Given an integer $l>0$, it is possible to choose coordinates $\left\langle z_{\alpha}, w_{\alpha}\right\rangle$ in neighborhoods $U_{\alpha}$ covering $\Delta^{\prime}$, so that $\left\langle z_{\alpha}, w_{\alpha}\right\rangle$ and $\left\langle z_{\beta}, w_{\beta}\right\rangle$ match to order l in $U_{\alpha} \cap U_{\beta}$.

Proof. The case $l=1$ is just Lemma 1 . We proceed by induction, assuming the lemma true for $l-1$, and suppose we have chosen coordinates which match to order $l-1$ for some finite covering $\left\{U_{\alpha}\right\}$ of $\Delta^{\prime}$. In $U_{\alpha} \cap U_{\beta}$,

and

$$
z_{\alpha}=z_{\beta}+\sum_{I} A_{\alpha \beta I}\left(z_{\beta}\right)\left(w_{\beta}\right)^{I}+O\left(w_{\beta}^{l+1}\right)
$$

$$
w_{\alpha}=w_{\beta}+\sum_{I} B_{\alpha \beta I}\left(z_{\beta}\right)\left(w_{\beta}\right)^{I}+O\left(w_{\beta}^{l+1}\right),
$$

where the sums are taken over all multi-indices $I$ of order $l$. Since, for $l>1$, we have $A_{\alpha \beta I}=-A_{\beta \alpha I}, \quad A_{\alpha \gamma I}=A_{\alpha \beta I}+A_{\beta \gamma I}, \quad B_{\alpha \beta I}=-B_{\beta \alpha I}$, and $B_{\alpha \gamma I}=B_{\alpha \beta I}+B_{\beta \gamma I}$, the $A_{\alpha \beta I}$ and $B_{\alpha \beta I}$ define one-dimensional cocycles on the covering $\left\{V_{\alpha}\right\}$, with coefficients in the sheaf $\mathcal{O}_{\Delta}^{q}$, for suitable $q$. Since $H^{1}\left(\Delta^{\prime}, \mathcal{O}^{q}\right)=0$, we can find holomorphic vectors $A_{\alpha I}(z)$ and $B_{\alpha I}(z)$ in $V_{\alpha}$, so that $A_{\alpha \beta I}=A_{\alpha I}-A_{\beta I}$ and $B_{\alpha \beta I}=B_{\alpha I}-B_{\beta I}$. In $U_{\alpha}$, define new functions by

$$
\tilde{z}_{\alpha}=z_{\alpha}-\sum_{I} A_{\alpha I}\left(z_{\alpha}\right) w_{\alpha}^{I} \quad \text { and } \quad \tilde{w}_{\alpha}=w_{\alpha}-\sum_{I} B_{\alpha I}\left(z_{\alpha}\right) w_{\alpha}^{I} \text {. }
$$

Then the functions $\left\langle\tilde{z}_{\alpha}, \tilde{w}_{\alpha}\right\rangle$ are coordinates in some neighborhood of $\bar{V}_{\alpha}$, and we may choose new coordinate neighborhoods $\tilde{U}_{\alpha}$, with $\tilde{U}_{\alpha} \cap \Delta^{\prime}=V_{\alpha}$. The new coordinates now match to order $l$, proving the lemma.

We now return to the domain $D \subset \Delta^{\prime}$ and cover the image of $\bar{D}$ by a finite number of coordinate neighborhoods $U_{\alpha}$ so that the coordinates $\left\langle z_{\alpha}, w_{\alpha}\right\rangle$ match to the third order. Let $\left\{\eta_{\alpha}\right\}$ be nonnegative $C^{\infty}$-functions with the support of $\eta_{\alpha}$ contained in $U_{\alpha}$, such that $\sum \eta_{\alpha}$ is identically 1 on a neighborhood of $f[\bar{D}]$. We define functions on $M$ by

$$
u^{i}=\sum_{\alpha} \eta_{\alpha} z_{\alpha}^{i}, \quad v^{i}=\sum_{\alpha} \eta_{\alpha} w_{\alpha}^{i} .
$$


Then $u^{i}$ and $v^{i}$ are everywhere defined differentiable functions, and in $U_{\alpha}$ we have

$$
\begin{aligned}
& u^{i}-z_{\alpha}^{i}=\sum_{\beta} \eta_{\beta}\left(z_{\beta}^{i}-z_{\alpha}^{i}\right)=O\left(w_{\alpha}^{4}\right), \\
& v^{i}-w_{\alpha}^{i}=\sum_{\beta} \eta_{\beta}\left(w_{\beta}^{i}-w_{\alpha}^{i}\right)=O\left(w_{\alpha}^{4}\right) .
\end{aligned}
$$

Hence $\langle u, v\rangle$ are differentiable coordinates in a neighborhood $O$ of $f[\bar{D}]$, and we can reduce $O$ so that the image of $O$ under $\langle u, v\rangle$ is $D \times B_{\delta}$, where $B_{\delta}$ is the ball $\sum\left|v_{i}\right|^{2}<\delta$. Set $\rho^{2}=\sum\left|v^{i}\right|^{2}$. Then in $U_{\alpha} \cap O$ we have

$$
\left|u^{i}\right|^{2}-\left|z_{\alpha}^{i}\right|^{2}=O\left(\rho^{4}\right), \quad\left|v^{i}\right|^{2}-\left|w_{\alpha}^{i}\right|^{2}=O\left(\rho^{5}\right) .
$$

Let $\phi$ be the convex function on $\Delta^{k}$ introduced at the beginning of the proof, and define a $C^{\infty}$-function $\psi_{\lambda}$ on $D \times B_{\delta}$ by

$$
\psi_{\lambda}(u, v)=\phi(u)+\lambda^{-2} \sum\left|v^{i}\right|^{2} .
$$

Then on $U_{\alpha} \cap O$, we have

$$
\psi_{\lambda}(u, v)-\psi_{\lambda}\left(z_{\alpha}, w_{\alpha}\right)=O\left(\left|u^{i}\right|^{2}-\left|z_{\alpha}^{i}\right|^{2}\right)+\lambda^{-2} O\left(\left|v^{i}\right|^{2}-\left|w_{\alpha}^{i}\right|^{2}\right),
$$

and so the Hessian of the difference with respect to the coordinates $\left(z_{\alpha}, w_{\alpha}^{\prime}\right)$ is $O\left(\rho^{2}\right)+\lambda^{-2} O\left(\rho^{3}\right)$ if $\lambda<1$. The Hessian of $\psi_{\lambda}\left(z_{\alpha}, w_{\alpha}\right)$ is a diagonal matrix, whose entries are $c\left(1+\left|z_{\alpha}^{i}\right|^{2}\right)\left(1-\left|z_{\alpha}^{i}\right|^{2}\right)^{-3}$ and $\lambda^{-2}$. Thus this Hessian is a positive definite matrix larger than $c I$.

Let $O_{\lambda}$ be the subset of $O$, where $\psi_{\lambda}(u, v)<1$. Then $\bar{O}_{\lambda} \subset O$ if $\lambda<\delta$. In $\bar{O}_{\lambda} \cap \bar{U}_{\alpha}$, the Hessian of $\psi_{\lambda}(u, v)$ with respect to $\left\langle z_{\alpha}, w_{\alpha}\right\rangle$ is a matrix which is greater than $c I+O\left(\rho^{2}\right)+\lambda^{-2} O\left(\rho^{3}\right)$. But in $\bar{O}_{\lambda}$, we have $\rho \leqq \lambda$, and so the Hessian is greater than $c I+O(\lambda)$. Thus there is a $\lambda_{\alpha}$ such that $\psi_{\lambda}(u, v)$ is strictly pluri-subharmonic in $\bar{O}_{\lambda} \cap U_{\alpha}$ for $\lambda<\lambda_{\alpha}$. Choose $\lambda=\min _{\alpha} \lambda_{\alpha}$. Then $\psi_{\lambda}(u, v)$ is strictly pluri-subharmonic in $\bar{O}_{\lambda}$.

If we set $\phi=\left(1-\psi_{\lambda}\right)^{-1}$, then $\phi$ is a pluri-subharmonic $C^{\infty}$-function in $O_{\lambda}$, and $\{p: \phi(p) \leqq c\}$ is a compact subset of $O_{\lambda}$ for each real $c$. It thus follows [3, Theorem 5.2.10] that $O_{\lambda}$ is a Stein manifold. Since $f[D] \subset O_{\lambda}$ and $\bar{\Delta}_{r}^{k} \subset D$, we have proved the following lemma:

LeMma 3. Let $f$ be a holomorphic embedding of the unit polydisk $\Delta^{k}$ into $M$. Then given $r<1$, there is a convex domain $D \supset \bar{\Delta}_{r}^{k}$ and an open subset $O$ of $M$, which contains $f[D]$ and is Stein.

We now use the fact that, if $f$ is an embedding of a Stein manifold $D$ into a Stein manifold $O$, there is a neighborhood $U$ of the zero section of the normal bundle of the embedding and an immersion $F: U \rightarrow O$, such that $F$ restricted to the zero section is $f$ (cf. [1, Hilfsatz 11]). Since $D$ is contractible and Stein, the normal bundle is equivalent to $D \times C^{n-k}$, 
and any neighborhood of the zero section must contain $\Delta_{r}^{k} \times \Delta_{\varepsilon}^{n-k}$ for some sufficiently small $\varepsilon$. Since $F=f$ and is one-to-one on $\Delta_{r}^{k} \times\{0\}$, we may take $\varepsilon$ small enough so that $F$ is one-to-one on $\Delta_{r}^{k} \times \Delta_{\varepsilon}^{n-k}$. By a change of scale in the last $n-k$ variables, we may replace $\Delta_{r}^{k} \times \Delta_{\varepsilon}^{n-k}$ by $\Delta_{r}^{k} \times \Delta^{n-k}$. This completes the proof of the proposition.

We now remove the restriction that $f$ is an embedding.

Proposition 2. Let $f$ be a holomorphic map of the $k$-dimensional unit polydisk $\Delta^{k}$ into an $n$-dimensional complex manifold $M$, and suppose that $f$ is regular at 0 . Then, given $r<1$, there is a map $F$ of $\Delta_{r}^{k} \times \Delta^{n-k}$ into $M$, which is regular at 0 and whose restriction to $\Delta_{r}^{k} \times\{0\}$ is $f$.

Proof. Let $g$ be the mapping of $\Delta^{k}$ into $\Delta^{k} \times M$, given by $g(z)=$ $\langle z, f(z)\rangle$. Then $g$ is an embedding. Hence by Proposition 1 , there is an embedding $G$ of $\Delta_{r}^{k} \times \Delta^{n}$ into $\Delta^{k} \times M$, which agrees with $g$ on $\Delta_{r}^{k} \times\{0\}$. Let $\pi: \Delta^{k} \times M$ be projection onto the factor $M$. Then $\pi \circ g=f$. Since $f$ is regular at 0 , we can choose an $(n-k)$-dimensional linear subspace $S$ of $\Delta^{n}$, so that the map $\pi \circ G$ restricted to $\Delta_{r}^{k} \times S$ is regular at 0 . Since $S$ contains an $(n-k)$-dimensional polydisk $\Delta^{n-k}$ about 0 , which we can take to be a unit polydisk by a change of scale, the proposition is established if we take $F$ to be the restriction of $\pi \circ G$ to $\Delta_{r}^{k} \times \Delta^{n-k}$.

Proposition 3. Let $f$ be a holomorphic immersion of the $k$-dimensional polydisk $\Delta^{k}$ into the complex manifold $M$. Then, given $r<1$, there is an immersion $F$ of $\Delta_{r}^{k} \times \Delta^{n-k}$ into $M$, whose restriction to $\Delta_{r}^{k} \times\{0\}$ is $f$.

Proof. The proposition is an immediate consequence of Proposition 1 and the following standard lemma:

LEMMA 4. Let $f$ be an immersion of a $k$-dimensional complex manifold $V$ into an n-dimensional complex manifold $M$. Then there is an $n$-dimensional complex manifold $N$, an embedding $h$ of $V$ into $N$, and an immersion $g$ of $N$ into $M$ such that $f=g \circ h$.

\section{BIBLIOGRAPHY}

1. O. Forster and K. J. Ramspott, Analytische Modulgarben und Endromisbündel, Invent. Math. 2 (1966), 145-170. MR 36 \#1702.

2. H. Grauert, Analytische Faserungen über holomorphvollständigen Räumen, Math. Ann. 135 (1958), 263-273. MR 20 \#4661.

3. L. Hörmander, An introduction to complex analysis in several variables, Van Nostrand, Princeton, N.J., 1966. MR 34 \#2933.

4. H. L. Royden, Remarks on the Kobayashi metric, Proc. Maryland Conf. on Several Complex Variables, Lecture Notes in Math., vol. 185, Springer-Verlag, Berlin and New York.

Department of Mathematics, Stanford University, Stanford, California 94305 